\title{
Sondage du Glacier par la méthode sismique
}

\section{Sub-surface glacier investigations: seismic soundings}

\author{
PAR A. SUSSTRUNK, ZURICH *
}

English synopsis p. 219

\section{I. - La méthode}

Dans ses grandes lignes, le procédé sismique est très simple: on provoque à la surface du glacier des explosions qui engendrent des perturbations, lesquelles se propagent sous forme d'ondes ćlastiques, parmi lesquelles on ne considère que les plus rapides, c'est-à-dire les ondes longitudinales (ondes de condensation et de (ilatation). Les ondes qui se propagent le long de la surface sont appelées « ondes directes». D'autres descendent an fond et sont réfléchies par la surface du bed-rock, ce sont les ondes réfléchies. Enfin, certaines ondes longitudinales, dont les rayons arrivent à l'inter-surface glacerocher sous un certain angle d'incidence (angle de la réflexion totale), cheminent dans le milieu inférieur, le long de cette inter-surface; une
Fig. 1. - On vort sur ha migue 1 a hes mégions

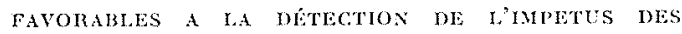
ONDES RÉrLECHES RT RËRACTÉS.

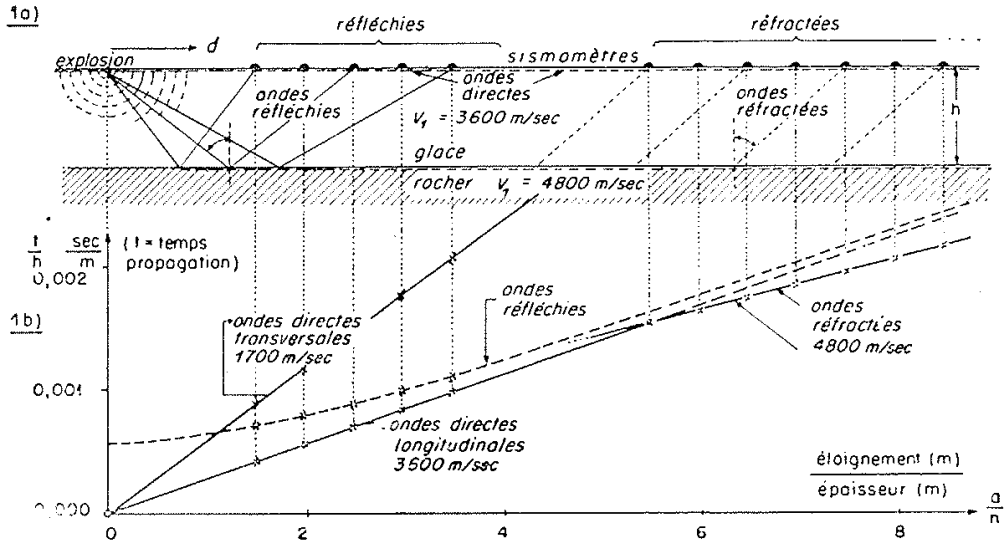

partie de leur énergie est émise continuellement vers le milieu supérieur, sous le même angle limite. Ces ondes, qui se propagent suivant la ligne brisée de la figure 1 , se nomment ondes réfractées.

Les données essentielles sont : les coordonnées relatives des explosions et des sismomètres, les temps de propagation des ondes directes, réfléchies et, éventuellement, des ondes réfractées; le quotient entre les ondes de propagation directe (longitudinales et transversales)

(*) Associc dans Dr H. Kneche et A. Süsstrunk, Zurich 53, Recherches géotechniques et géophysiques. et les éloignements des sismomètres correspondants foumissant les vitesses de propagation des ondes dans la glace: $v_{1}=3.600 \mathrm{~m} / \mathrm{s}$ et $v_{\mathrm{t}}=1.700 \mathrm{~m} / \mathrm{s}$ environ. Le produit de la vitesse $v_{1}$ et du temps de propagation correspondant à une onde réfléchie est égal au chemin total de cette onde dans la glace. On en tire aisément l'épaisseur du glacier, dans le cas d'une inter-surface glace-bed-rock parallèle à la surface de la glace.

Un sismometre situé sur le glacier, à quelques centaines de mètres du point d'explosion, devrait indiquer, en principe, l'arrivée de toutes les ondes définies ci-dessus. En réalité, les 

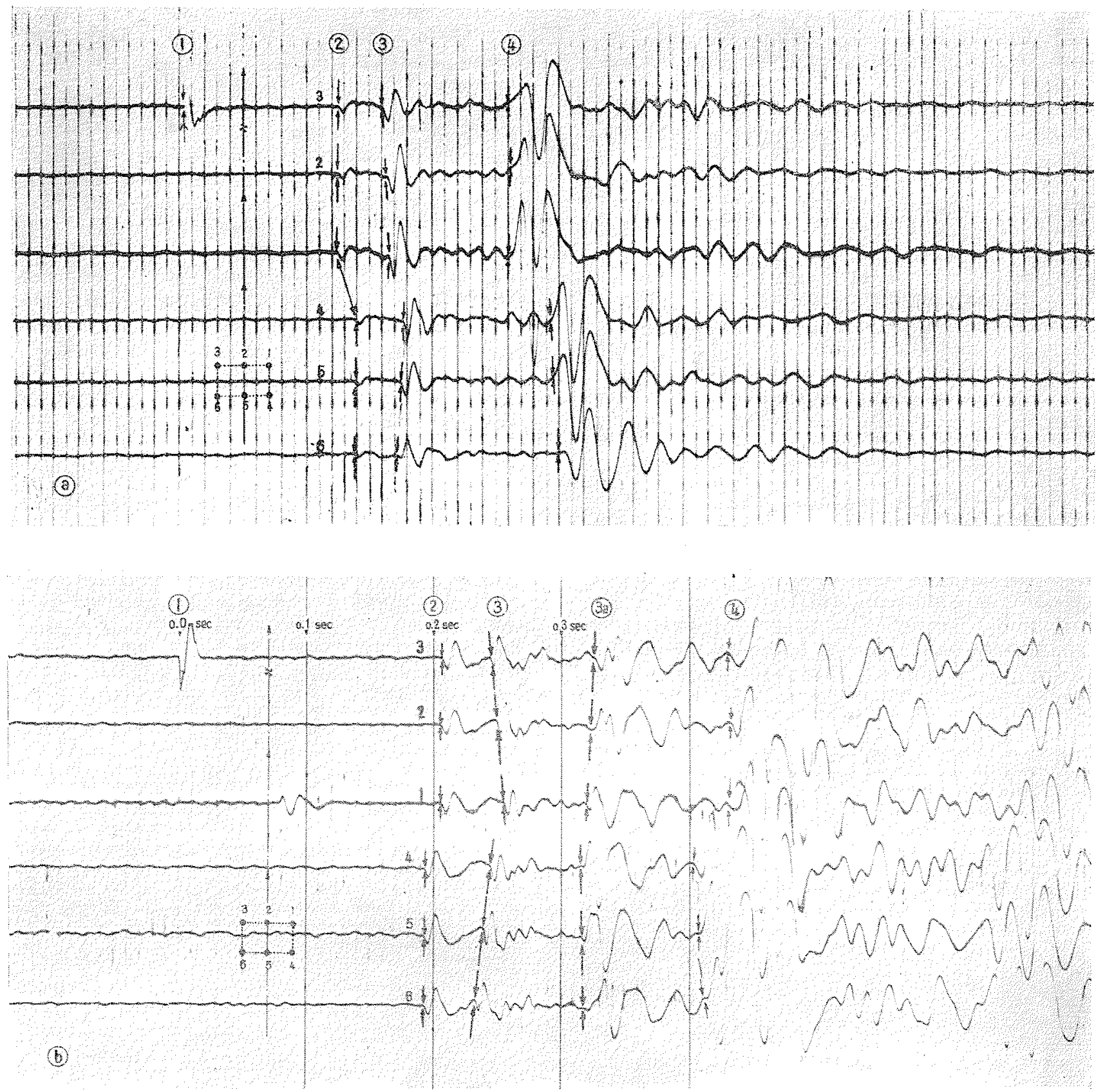

Fig. 2. - Sismogramies.

1. Instant de lexplosion;

2. Arrive de l'onde directe longitudinale;
3, 3 a. Arrivé d'ondes réflechies par le bedrock: 4. Arrive de l'onde directe transversale. impetus des ondes considérées ne sont pas observables pour des cloignements quelconques. Pour pouvoir bien constater l'arrivée du front de l'onde réfléchie (c'est-à-dire pour qu'elle se distingue nettement des perturbations produites par les ondes directes), il faut choisir l'éloignement des sismomètres de telle sorte que l'onde réfléchie arrive entre les deux ondes directes: longitudinale et transversale. Puisque, dans la glace, la vitesse de propagation de l'onde trans- rersale est environ la moitié de celle de l'onde longitudinale, la condition ci-dessus conduit à fixer l'éloignement des sismomètres par rapport au point d'explosion entre deux limites qui sont 1,2 à $4 h$ environ, $h$ désignant l'épaisseur de la glace.

$\mathrm{Si}$ on considire les ondes réfractées, on choisit, en général, des éloignements tels que ces ondes arrivent arant les ondes directes. Cependant, sur la glace - par suite de la faible diffé- 
rence entre les vitesses de propagation dans la glace et dans le rocher - les éloignements des sismomètres par rapport au point d'explosion deviennent très grands (5 à $8 h$ cnviron). Ceci explique que, sur la glace, on utilise plutôt la sismique par réflexion que la sismique par réfraction.

La figure 1 a représente le trajet des ondes dans le cas le plus simple d'une couche de glace à surface parallèle au fond rocheux.

La figure $1 b$ représente les hodographes des différentes ondes.

Des considérations d'ordre théorique concernant le bilan énergétique [1] de la réflexion des ondes élastiques dans la discontinuité glace/rocher imposent plutôt un rapport $d / h$ inféricur à 2 , avec un maximum de second ordre dans la région $d / h=1,5$, dont, cependant, on ne peut profiter que dans le cas de profondeurs de la glace dépassant $250 \mathrm{~m}$. Dans le domaine $d / h>2$, le bilan énergétique, devenant défavorable, cause l'emploi de quantités d'explosif considérablement augmentées.

La figure 2 représente deux sismogrammes fournissant des ondes réfléchies de très bonne qualité. Le deuxième possède même deux réflexions très prononcées. Les détails très importants de chaque sismogramme sont: l'instant de l'explosion (voir première trace) et le contrôle du temps (commandé par un diapason de 100 périodes qui ouvre et ferme un écran à fente).

En général, J’onde directe longitudinale apparait beaucoup. plus faiblement sur le sismogramme que l'onde réfléchie; ceci est dû à la sensibilité uniquement verticale des sismometres utilisés. Si l'on enregistrait les amplitudes totales, celle de l'onde primaire serait einq à dix fois plus grande que celle de l'onde réfléchic. Ajoutons que, d'une façon générale, la qualití des sismogrammes obtenue est beaucoup moins bonne que celle de ces deux exemples et qu'une certaine expérience est nécessaire à l'interprétation des enregistrements.

\section{II. - L'élaboration}

Le lieu géométrique de tous les points de réflexion correspondant au temps de propagation d'une onde réfléchie est constitué par un ellipsoïde de révolution dont les foyers coïncident avec le point d'explosion d'une part, et l'emplacement du sismomètre considéré d'autre part. Pour déterminer dans l'espace la position du miroir réfléchissant (surface du bed-rock), il faut trouver le plan tangent aux trois ellipsoïdes dont les foyers sont constitués respectivement par un point d'explosion et par trois sis- momètres placés en triangle. Ceci suppose que la surface du bed-rock est plane sur une superficie de 400 à $1.000 \mathrm{~m}^{2}$ au moins. La solution analytique de ce problème est très laborieuse, et nous utilisons un procédé graphique que nous avons mis au point nous-mêmes. Ce « principe de trois points $»$ a été introduit par A. KRers en $1936^{*}$; la figure 3 montre l'importance de ce procédé : la position des éléments réfléchissants du bedrock peut différer considérablement de l'endroit du « profil » sismique. La figure 3 montre également la disposition des sismomètres par rapport aux points d'explosion (« profil » sismique) dans nos sondages des glaciers, ainsi que la représentation des résultats; on obtient à la fois l'emplacement et l'orientation, dans l'espace, de l'élément réfléchissant (cote, position en projection dans une coupe horizontale et direction de la pente maxima du plan réfléchissant).

L'examen rapide d'un sismogramme donne tout de suite quelques renseignements purement

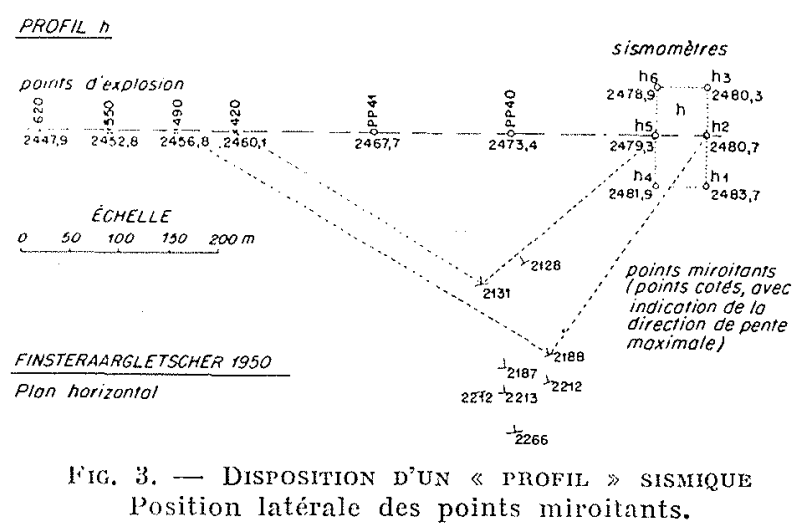

qualitatifs sur la position des éléments réfléchissants. Il est évident qu'en regardant, par exemple, la première réflexion du sismogramme 2 (fig. 2), les sismomètres 1 et 4 sont situés plus loin de la surface du bed-rock sous-glaciaire que les points 3 et 6 , c'est-à-dire que l'onde réfléchie arrive đu côté gauche; la deuxième réflexion, au contraire, du côté droit par rapport à la direction du profil.

De plus, on peut ajouter à l'élaboration géométrique des considérations énergétiques : la variation des amplitudes de l'onde réfléchie en fonction de l'angle d'incidence est gouvernée par les constantes élastiques et les densités de la slace et du matériau constituant le miroir réfléchissant. En général, on n'a pas besoin d'examiner ainsi indirectement les propriétés du bedrock. Toutefois, on rencontre assez souvent des

(*) Des solutions semblables ont été publiées par F. Gassmann [2] et S. J. Pirson [3]. 
réflexions produites par des discontinuités encore inexplorées dans l'intérieur du glacier. Là où l'on réussit à étudier les variations énergétiques des réflexions correspondant à un tel horizon, il est possible d'obtenir indirectement quelques renseignements sur l'état mécanique de ces miroirs internes. Malheureusement, ces études sont très délicates, et leur mise au point n'est pas assez avancée pour fournir actuellement des résultats. En Suisse, les dissipateur's du glacier du Mont-Collon et du glacier inférieur de l'Aar présentent ces anomalies, et l'on soupconne qu'il s'agit de moraines internes, sous forme d'inter-surface plus ou moins déformées entre des couches de glace d'origine différente.

Différentes sources d'erreurs entachent la méthode : d'une part, des erreurs lices aux procédés techniques (topographie, mesure des temps de propagation, procédé des trois points, élaboration graphi(que), conduisant, selon notre expérience, à des erreurs de l'ordre de $1 \%$; d'autre part, des irrógularités des milieux et du lit sousglaciaire, qui influent sur la qualité des sismogrammes, portant la limite d'erreur à $\pm 3 \%$ environ.

Un certain contrôle est possible par la comparaison des profondeurs des éléments réfléchissants résultant de profils différents, mais qui tombent dans la même région : en général, les différences des profondeurs ne dépassent que rarement la limite de $\pm 3 \%$. De même, les résultats de seize forages thermiques exécutés sur le glacier du Gorner ont montré une concordance avec les résultats des sondages sismiques avec une erreur moyenne inférieure à $\pm 3 \%$.

Ce sont la valeur et la direction de la pente maxima de l'élément réfléchissant qui sont surtout influencées par les hypothèses du «procédé de trois points $»$, surtout au cas de pente faible. Aussi on ne donne généralement à ces indications qu'une valeur qualitative ef nous renonçons, en général, à indiquer la valeur de la pente.

\section{Limite d'application. - Les profondeurs son-} dées dans les glaciers alpins varient entre 80 et $500 \mathrm{~m}$. Il semble qu'il y ait, pour cette méthode, une limite d'application pour des épaisseurs inférieures à $50-100 \mathrm{~m}$. Il est vraisemblable que les raisons de l'existence d'une telle limite sont de nature glaciologique, car les couches d'épaisseur si réduites sont, en général, dans un état de détérioration avancé et ne se prêtent plus bien à l'application des procédés sismiques, soit parce que l'état de la glace est Ioin de celui d'un milieu homogène, soit parce qu'une couche de glace d'épaisseur de 50 à $100 \mathrm{~m}$ n'est pas assez plastique pour qu'un bon contact avec le rocher soit assuré.

\section{III. - Equipement-Personnel}

Jusqu'à la fin de l'année 1948, les recherches sismiques sur les glaciers, en Suisse, ont été exécutées à l'aide d'un sismographe transportable, du type mécanique-optique, qui est la propriété de la Commission Helvétique des Glaciers, et qui avait été construit par le Professeur A. KrEIs. Il s'agit d'un sismographe dit universel, qui enregistre le mouvement du sol suivant les trois composantes perpendiculaires. On espérait obtenir, par ce dispositif (en combinant les amplitudes de l'onde réfléchie suivant les trois composantes), l'angle d'incidence de l'onde réfléchie, et faciliter ainsi la détermination de la position de la surface réfléchissante dans l'espace. Ce but n'a pas été atteint, à cause de la faible sensibilité de l'équipement et de certaines irrégularités dans l'ajustement du sismographe.

La méthode de travail avec cet appareil était différente de celle susmentionnée; le sismographe restait stationnaire, et c'était le point d'explosion qui se déplaçait le long du profil avec, également, des points latéraux.

Depuis 1949, nous utilisons un équipement moderne avec sismomitres électriques et amplification électronique. Il s'agit d'un appareil d'origine américaine (Century-Tulsa), dont nous n'utilisons sur le glacier que six canaux, selon la disposition représentée sur la figure 3 . Le poids des appareils est inférieur à $200 \mathrm{~kg}$ (sismomètres, circuits de contrôle, amplificateurs, oscillographe, chambre noire, accumulateurs, câbles de communication entre sismometre et station d'enregistrement). Il faut y ajouter le poids d'accessoires divers $(200$ a $300 \mathrm{~kg}$ : ontils pour forer des trous de mine - un mètre de profondeur, $35 \mathrm{~mm}$ de diamètre --, câbles pour la communication téléphonique, explosifs, amorces électriques, instruments de topographie, groupe électrogène pour charger les accumulateurs, tentes, cacolets, matériel de réserve, etc.), soit au total un poids de 400 à $500 \mathrm{~kg}$. La charge partielle la plus lourde est environ de $35 \mathrm{~kg}$; e'est done un équipement facilement transportable, et bien qu'il ait été construit pour la géophysique du pétrole, il a fait ses preuves sur les glaciers à notre entic̀re satisfaction. Cet appareil est très sensible (le mouvement du terrain est enregistré avec un agrandissement de l'ordre de 1 million). Quant aux quantités d'explosifs comparées à celles utilisées avec l'ancien équipement, elles se trouvent réduites de 80 à $90 \%$. Actuellement, nous employons 50 à $200 \mathrm{~g}$ de dynamite gélatinée pour des trajets de 300 à 1.000 mètres.

L'équipe nécessaire pour la conduite de ces sondages sismiques comprend 8 hommes, dont 4 manœuvres:

- 1 chef dirigeant les travaux et soccupant 
sur place de l'élaboration de résultats provisoires;

- 1 opérateur qui manœuvre les appareils (enregistrement, développement des films);

- 1 shooter qui implante les forages, place les sismomètres et commande les explosions;

- 1 topographe qui implante et lève à I'avance les points d'explosion et les emplacements de sismomètres;

- 4 aides : 1 aide topographe, 1 à 2 aides foreurs; 1 à 2 aides chargés de préparer les mines, de placer les instruments, etc.

Dans des conditions favorables, on peut obtenir dans une journée 30 à 35 sismogrammes, correspondant à 5 ou 7 profils (voir fig. 3), c'est-àdire des données suffisantes pour élaborer une centaine d'éléments réfléchissants du lit sousglaciaire. Quant à l'élaboration (topographie superficielle y comprise), il faut compter, pour 30 éléments réfléchissants, un jour de travail pour deux ingénieurs ou techniciens expérimentés. Si les conditions ne sont pas bonnes et les données sismiques difficiles à interpréter, le délai est augmenté considérablement.

Un sondage complet d'une région de glacier se compose d'une série de systèmes élémentaires (profils) identiques à ceux de la figure 3, dispo- sés de telle manière que les points réfléchissants du lit sous-glaciaire qui en résultent donnent la topographie du bed-rock d'une manière satisfaisante (voir fig. $6 \mathrm{~b}$ ). Mais s'il ne s'agit pas des dissipateurs, dont la largeur est grande par rapport à l'épaisseur, il est souvent très difficile d'obtenir des éléments réfléchissants dans toute la zone prospectée. Aux difficultés géométriques (profils transversaux pas assez longs) se superposent les difficultés d'exécution du travail dans les régions erevassées le long des bords, etc., d'où ne résultent souvent que des sismogrammes de mauvaise qualité.

\section{IV. - Glaciers européens sondés par procédés sismiques}

La liste ci-après constitue un résumé du développement historique de l'application des méthodes sismiques aux sondages glaciaires. C'est essentiellement sous l'impulsion de MM. Motres et Krars que ce développement s'est amplifié. Les publications de Motres $[4,5]$ contiennent déjà presque toutes les considérations essentielles sur ces méthodes. Les chiffres entre crochets [] réfèrent à la bibliographie ci-annexée (1).

\section{LISTE DES GLACIERS}

\begin{tabular}{|c|c|c|}
\hline ANNÉES & Glaciers & AUTEURS \\
\hline $\begin{array}{c}1926-28 \\
1929 \\
1929 \\
1932 \\
1935 \\
1936-37-38-39- \\
47-48-50 \\
1943 \\
1946 \\
1947 \\
1948 \\
\\
1948 \\
1949 \\
1949 \\
1949 \\
1949-50\end{array}$ & 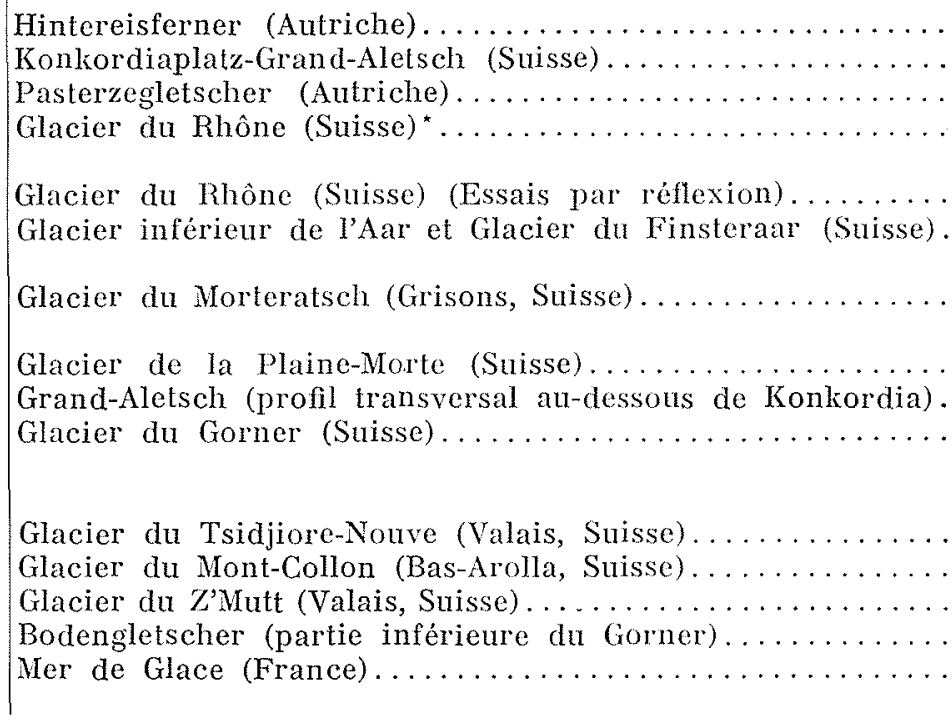 & 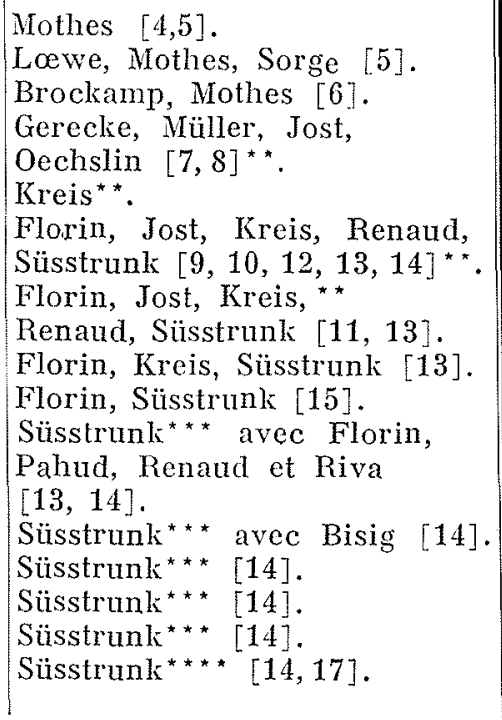 \\
\hline
\end{tabular}

(1) Seulement après avoir terminé ce mémoire l'auteur a eu connaissance que quelques essais par réflexion sismique ont été exécutés sur des glaciers en Norvège (Hardangerjökul) et en Suède (glacier de Kebnekaise) en 1947 ou 1948 environ. La publication [18] qui mentionne ces études ne contient pas de détails.

(*) Mèthode sismique par réfraction.

(*) Recherches scientifiques exécutées au nom de la
Commission Helvétique des Glaciers (les sondages sur le glaciel inférieur de l'Aar ont été subsidés par les Kraftwerke Oberhasli A.G. Innertkirchen).

$\left(^{\star \star *}\right)$ Sondages exécutés sur l'ordre et aux frais de l'EOS, Lausanne, pour Grande-Dixence S.A., par l'entreprise Dr H. KNechT et A. Süsstrove, Zurich.

$\left(\star_{\star \star \star}\right)$ Sondage exécuté sur l'ordre et aux frais du Services Etudes de la Région d'Equipement Hydraulique Alpes II de l'Electricite de France, par Dr H. KNechT et A. Süsstrunk, Zürich. 


\section{V. - Exemples}

a) Glacier du Gorner (Valais, Suisse).

Une surface de $7 \mathrm{~km}^{2}$ environ a été sondée en août et septembre 1948 (fig. 4). Equipement «Kreis ». A l'aide de 25 stations du sismographe et exviron 1.100 explosions, on a obtenu quelques 500 points de réflexion, qui fournis- saient les éléments de base pour une carte de bed-rock sous-glaciaire (fig. $4 a$ ).

De cette prospection, il résulte - en accord avec les observations glaciologiques superficielles [16] - que e'est le Grenzgletscher (y compris le Zwillinggletscher) (qui constitue le glacier principal parmi les aflluents du grand dissipateur du Gorner. La figure $4 b$ constitue une autre représentation des résultats, traduits en courbes d'égale profondeur de la slace.

Fig. 4 a. - Glacier du Gorner.

Topographie du bed-rock sous-glaciaire.
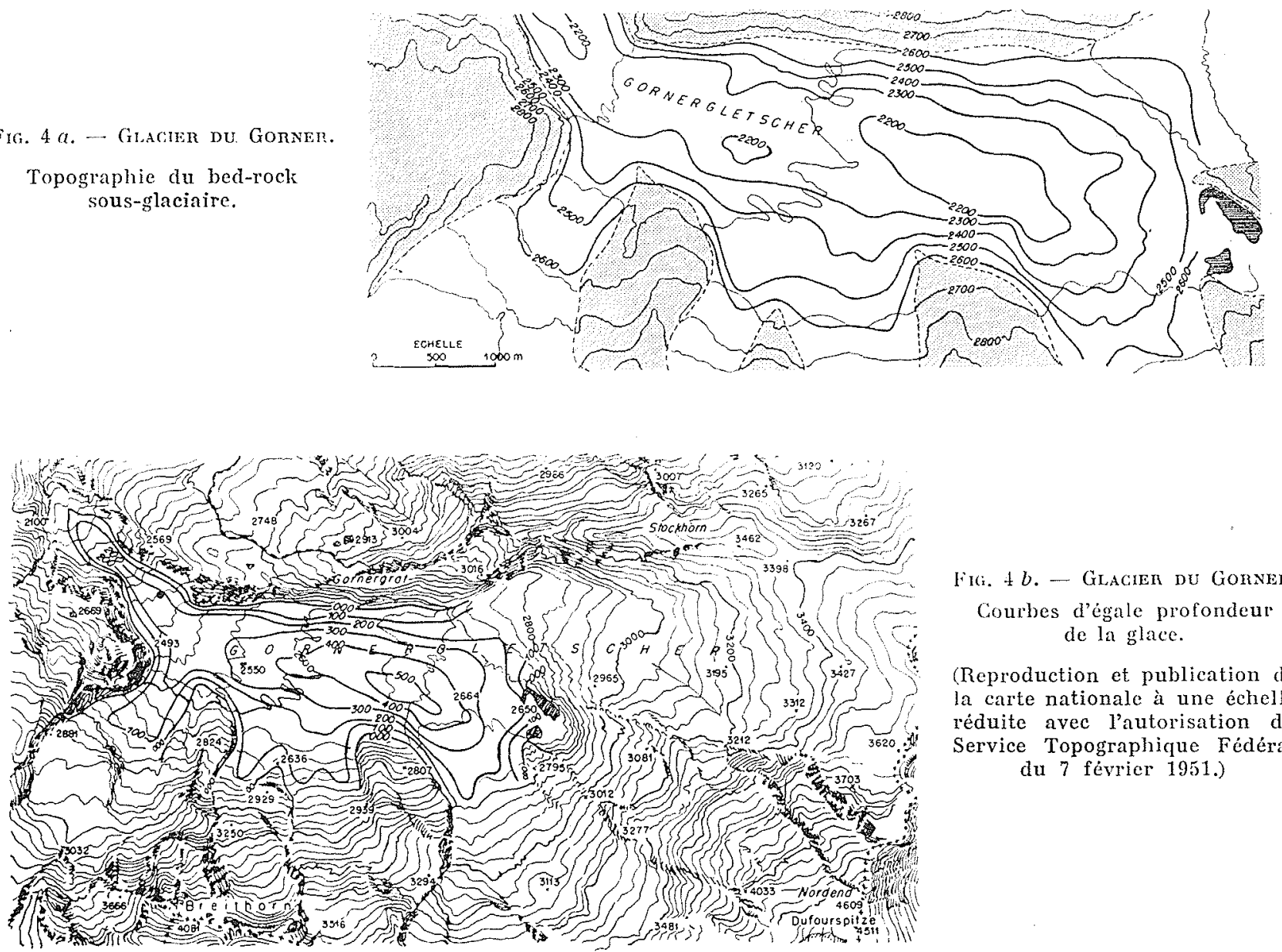

Fili, $4 b$. - Glacien du Gonner.

Courbes d'égale profondeur de la glace.

(Reproduction et publication de la carte nationale à une échelle réduite avec l'autorisation du Service Topographique Fédéral du 7 février 1951.)

Pour la première fois en Suisse, les sondages sismiques effectués sur le Gorner ont été contrôlés par des forages. L'EOS a fait exécuter une quinzaine de forages par le procédé Calcrati, lesquels ont montré une bonne concordance avec les profondeurs obtenues par sismique réflexion.

\section{b) Glacier du Mont-Collon (Valais, Suisse).}

$1 \mathrm{~km}^{2}$ environ a été sondé an début du mois de juillet 1949. Equipement «Century ». Sur la plupart des sismogrammes, on a observé deux réflexions, dont la première était, en général, faible et peu régulière. L'élaboration des résultats a fourni deux horizons réfléchissants plus ou moins parallèles; de même, des profils levés immédiatement à l'aval du glacier, à l'aide de la méthode sismique réfraction, ont donné des résultats étonnants. Ils ont montré, 200 à 300 mètres à l'aval de la langue terminale actuelle, la présence d'une conche de glace fossile d'une épaisseur de plusieurs dizaines de mètres, protégée par une couche d'alluvions de 4 à 10 mètres d'épaisseur. En mai 1949, l'EOS avait fait creuser une galerie, le long de la rivière sous-glaciaire (en agrandissant simplement la galerie naturelle creusée par le torrent lui-même). La figure 5 représente l'ensemble de 
ces observations : les figures $a$ et $b$ donnent les courbes de niveau des deux horizons mentionnés. Sur la figure $a$, on remarquera, en outre, la position de la galerie sus-indiquée; sur la figure $b$, on notera la position des coupes et des profils sismiques par réfraction exécutés à l'aval du glacier. Enfin, sur la figure $c$, on constate que le lit du torrent et la surface de la couche alluvionnaire coïncident parfaitement avec l'horizon supérieur.

Par contre, l'horizon inférieur semble correspondre avec le fond de la couche de glace fos- sile, d'âge et d'origine incertains, qui doit être séparé de la glace «vivante » par une moraine intermédiaire, ou peut-être seulement par une zone de glace contenant beaucoup de débris et de sables constituant un milieu donnant lieu a une réflexion partielle des ondes élastiques.

On n'a pas jugé opportun, jusqu'à présent, de contrôler les résultats de ces sondages par des forages; toutefois, pour vérifier indirectement l'existence de la glace fossile à $200 \mathrm{~m}$ à l'aval du dissipateur actuel, on a commencé des mesures de niveau précises sur ce terrain, la
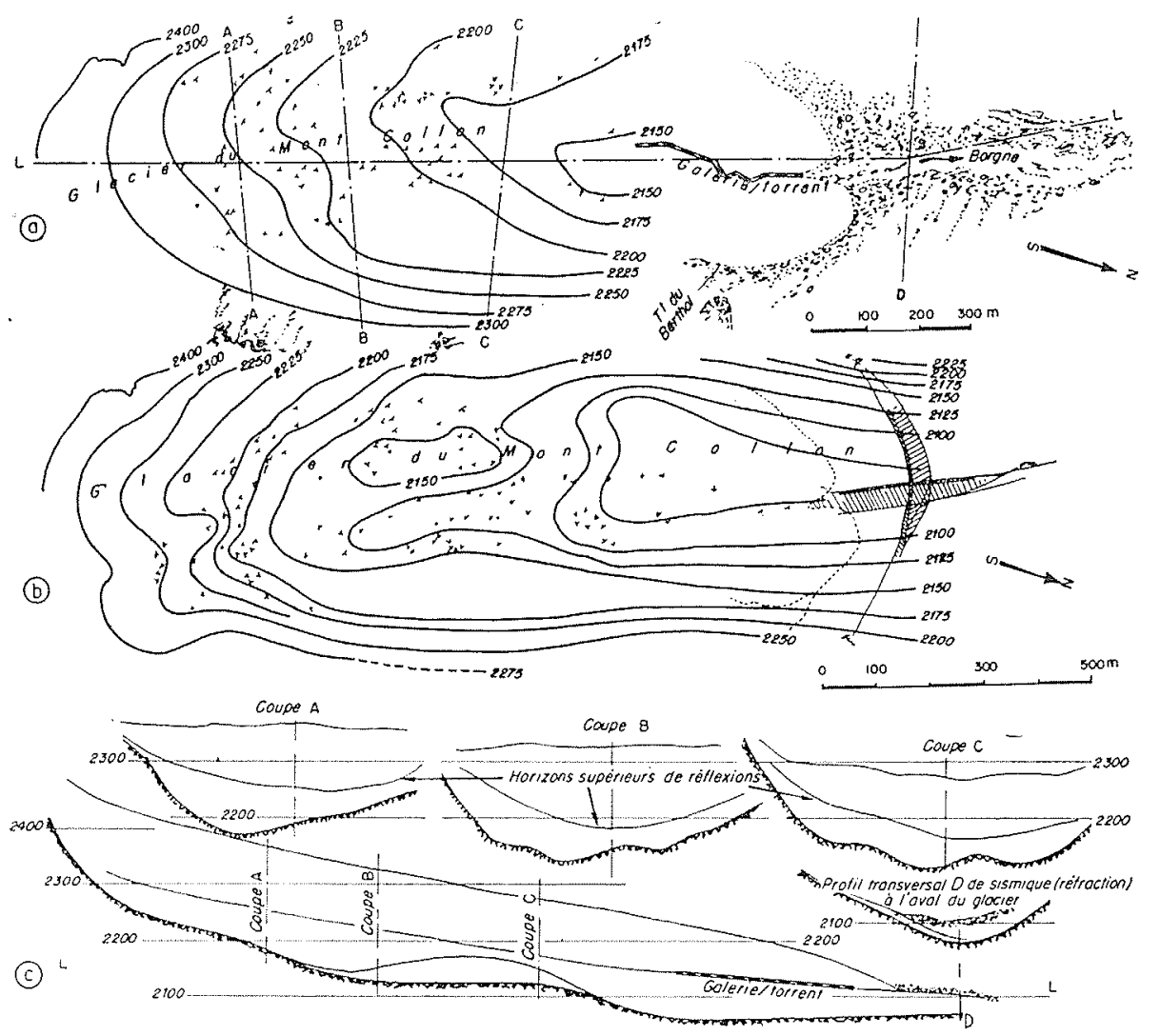

Fia. 5. - Résulatats du sondage sismique du gracier du Mont-Collon (1949).

(a) Topographic de l'horizon supérieur (moraine intermédiaire?).

(b) Topographie de l'horizon inférieur (bed-rock).

(c) Coupes transversales et coupe longitudinale.

fonte lente de la glace devant causer un abaissement d'au moins $1 \mathrm{~cm}$ par an; bien que les résultats des nivellements auxquels il a été procédé pendant l'année 1950 ne soient pas convaincants, ils fournissent, malgré tout, des indices corroborant l'existence d'une telle couche de glace fossile sous les alluvions.

De même que pour le Gorner, les sondages du glacier du Mont-Collon ont été exécutés à la demande et aux frais de la $\mathrm{S}$. A. l'Energie de l'Ouest-Suisse (EOS) à Lausanne, et ils constituent une partie des importantes études concernant le projet de la Grande-Dixence. c) Mrir id: Glace.

Des sondages sismiques - intéressant une surface de $1,6 \mathrm{~km}^{2}$ environ - ont également été exécutés pour le compte de l'Electricité de France sur la Mer de Glace, en mai 1950. Le but et les résultats de ces travaux sont exposés dans la communication de MM. Ract-Madoux et Reynaud [15].

Différant des glaciers sondés en Suisse, qui possèdent un lit en «U», la Mer de Glace présente une section en forme de « V très prononcée (voir fig. $6 \mathrm{c}$ ). Malgré l'inclinaison 


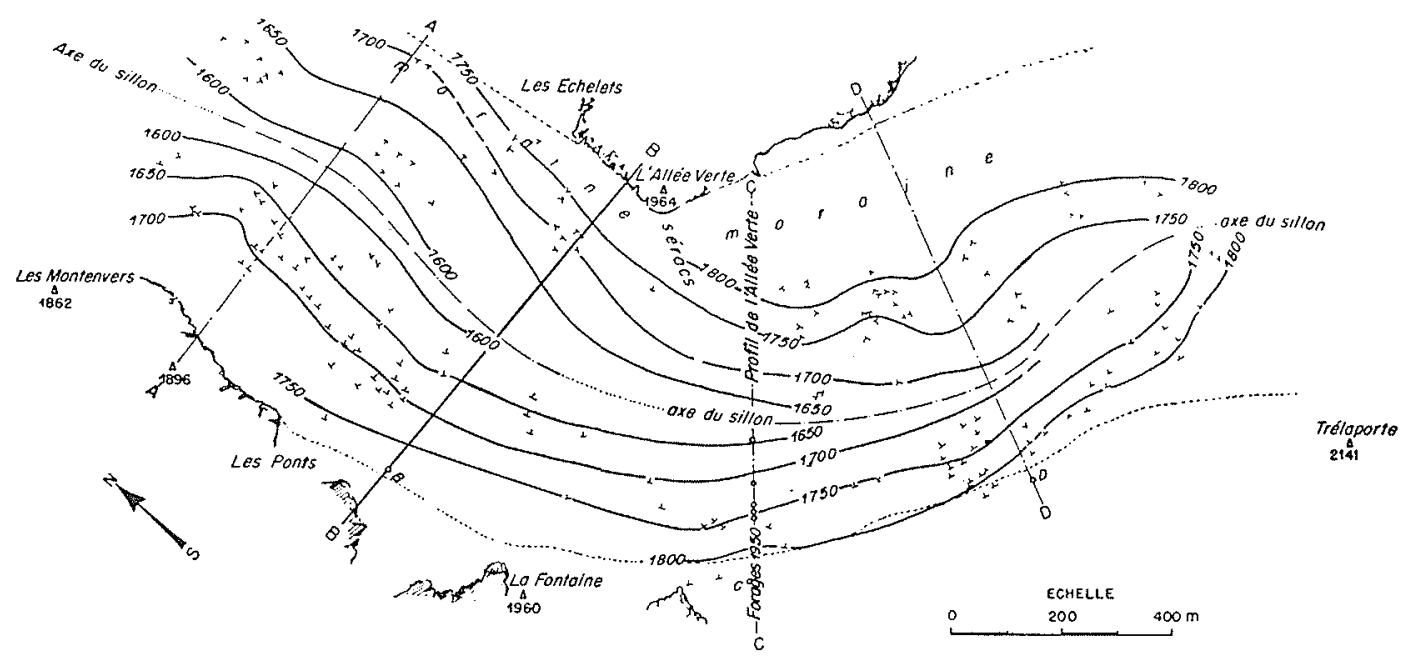

Frg. $6 a$. Sondages sismouts de la Mer de Glace en 1950. Résultats obtenus.

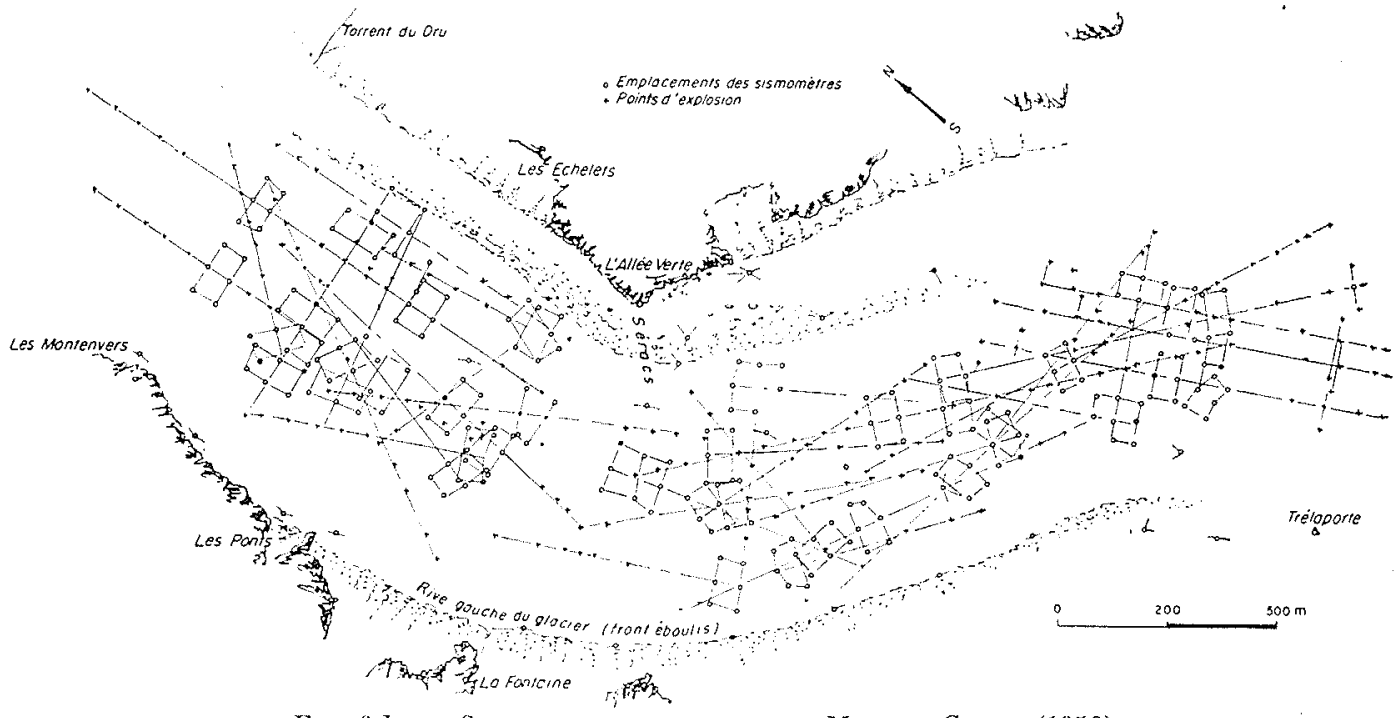

Fig. 6 b. - Sondaces sismigues de fa Mer de Glace (1950).

Plan des points levés.

des pentes du bed-rock, le procédé sismique réflexion s'est bien prêté aux sondages du lit de la Mer de Glace. La figure 6 a donne les résultats obtenus, la figure $6 \mathrm{~b}$ représente la position des points levés et celle des profils sismiques, ce qui, en outre, donnera une idée de l'importance du travail de topographie lié aux sondages sismiques des glaciers.

En certaines régions de la $\mathrm{Mer}$ de Glace, nous avons constaté une diminution de la vitesse des ondes directes, diminution que nous n'avions jamais rencontrée sur d'autres glaciers. Cette observation nous a fait soupconner que cette anomalie pouvait être causée par l'état de la glace, état influencé, dans certaines régions, par les déformations et pressions latérales con- sidérables. L'élaboration des résultats, à l'aide d'une valeur réduite de la vitesse $(3.500 \mathrm{~m} / \mathrm{s}$

\section{Coupe tronsversole $B-B$}

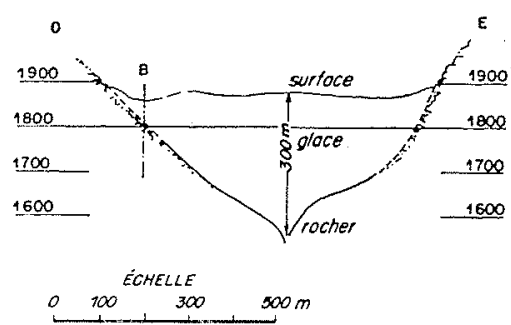

FIG. $6 c$.

Mer de Glace.

Coupe transversale de $\mathrm{BB}$.

en moyenne), donnait des résultats qui différaient très sensiblement de ceux obtenus par 
forage. Par contre, ceux calculés avec la valeur normale de $3.600 \mathrm{~m} / \mathrm{s}$ concordaient avec ces résultats. Si l'on étudie les variations de ces vitesses en fonction de la distance du point

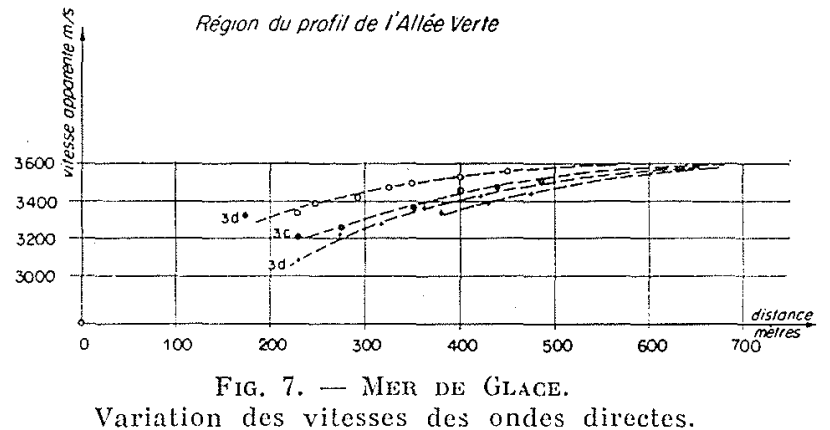

d'explosion au sismomètre, on obtient des diagrammes analogues à ceux de la figure 7, diagrammes sur lesquels nous avons représenté les variations de vitesse constatées les plus prononcées. Une explication de ce fait est fournie par la présence de crevasses étendues dans les régions de vitesses anormales; ces crevasses imposent aux ondes un trajet plus élevé, dont la différence, par rapport au trajet normal, diminue évidemment avec des distances croissantes.

\section{VI. - Remerciements}

Nous tenons ici à exprimer notre gratitude à la Société l'Energie de l'Ouest-Suisse, pour Grande Dixence S. A., et à l'Electricité de France de nous avoir autorisés à publier les résultats des travaux exécutés pour leur compte, à savoir : d'une part, la reproduction des plans relatifs aux glacier du Gorner et du Mont-Collon, et d'autre part, la reproduction des plans relatifs à la Mer de Glace.

\section{VII. - Bibliographie}

[1] BuvT (H.). - Ein Beitrag zur Theorie der Reflexion und Brechung elastischer Wellen an Unstetigkeitsflächen.

(Zeitschrift für Geophysik, 8 (1932), pp. 130-144 et $305-322$.)

[2] Gassmax (F.). - Seismische Bestimmung einer reflekticrenden Ebene.

(Beiträge zur angelvandten Geophysik, 7 (1937), pp. 17-20.)

[3] Prisor (S. J.). - Practical graphical and approximation Methods for Dip Shooting Calculations.

(The Oil Weekly (april 26, 1937), pp. 22-34.)
[4] Motrus (H.). - Seismische Dickenmessungs von Gletschereis.

(Zeitschrift fïr Geophysik, 3 (1927), 121-134.)

[5] Mothes (H.). - Neue Ergebnisse der Eisseismik. (Zeitschrift für Geophysik, 5 (1929), pp. 120-144.)

16] Brockamp (B.) et Mothes (H.). - Scismische Untersuchungen auf dem Pasterzegletscher.

(Zeitschrift für Geophysik, 6 (1930), pp. 482-500 et 7 (1931), 232-240.)

17] Grancke (F.). - Wellentypen, Strahlengang und Tiefenberechnung bei seismischen Eisdickenmessungen auf dem Rhonegletscher.

(Thèse Göttingen (1933). 28 pages; voir aussi : Zeitschrift für Geophysik, 8 (1932), pp. 65-71.)

[8] JosT (WV.). - Die seismischen Eisdickenmessungen auf dem Rhonegletscher 1931.

(Bericht der Gletscherkommission der Schueizerischen Naturforschenden Gesellschaft. Denkschriften des SNG, 71, Abhandlung 2, Zürich (1936).)

(9) Mercantor (P.-L.). - Les sondages séismométriques do la commission des glaciers à l'Unteraar.

(Actes de la Soc. Helvétique des Sciences Naturelles (1936), pp. 271-273.)

[10] KnErs (A.). - Ergebnisse der seismischen Eistiefenmessungen auf dem Unteraargletscher.

(Verhandl. der Schweiz, Naturforschenden Gesellschaft (1941), pp. 99-100.)

[11] Kreis (A.), - Seismische Sondierungen auf dem Morteratschgletscher.

(Verhandlungen der Schweiz. Naturforschenden Gesellschaft (1944), p. 95.)

[12] Marcanton (P.-L.). - Les sondages séismométriques de la commission helvétique des glaciers à l'Unteraar.

(Publication du Bureau Central Séismologique International, série A, Travaux Scientifiques, fasc. 16 (1946), pp. 52-53.)

[13] Rrnaud (A.) el Mercanton (P.-L.). - Les sondages sismique de la Commission Helvétique des glaciers. (Publications du Bureau Central Séismologique International, série A, Travaux Scientifiques, fasc. $17(1948)$. pp. $66-78$.

[14] Süssrrunk (A.). - - Seismische Messungen auf Gletschern (1948-1950). (Verhandlungen der Schweiz. Naturforschenden Gesellschaft (1950).)

[15] Haferi (R.) et Kasser (P.). - Beobachtungen im Firn- und Ablationsgebict des grossen Aletschsletschers.

(Mitteilung Nr. 15 aus der Versuchsanstalt für Wasserbau und Erdbau an der ETH in Zürich, et Schweizerische Bauzeitung, 66 (1948) Nr. 35;36.)

[16] Renaud (A.). - Notice sur le glacier du Gorner (Suisse).

(Mémoire p. r. à la Tournée Glaciologique 1950, 8 pages. Soc. Hydrotechnique de France, Paris $(1950)$.

[17] Ract-Madoux (X.) et Reynaud (L.). - Le sondage du glacier en profondeur : la Mer de Glace. (Mémoires et Travaux de la Société IIydrotechnique de Fronce. I (1951). La Houille Blanche A (1951).

[18] Hedström (H.) et Kollert (R.). - Seismic Sounding of Shallow Depths.

(Tellus, Stockholm, I (1949), No 4, pp. 24-36.) 


\section{I S C U S S I O N}

Se référant aux glaciers fossiles mentionnés par M. Susstrunk, M. Cherrey demande si ces masses glaciaires découvertes paraissent avoir participé au mouvement de l'ensemble du glacier.

M. Susstrunk précise qu'il s'agit probablement d'une masse de glace qui appartenait au glacier supérieur d'Arolla qui s'est retiré de cet endroit il y a une vingtaine d'années déjà. Sur cette masse de glace fossile, couverte de moraines, le glacier du Mont Collon probablement a avancé. Seulement maintenant, en régime de retraite, on peut supposer que la couche inférieure de glace participe en général au mouvement de l'ensemble du dissipateur de ce glacier.

M. le Président conclut que les deux masses glaciaires sont en mouvement restreint pour le résidu et intégral dans le glacier principal, sous la poussée du névé.

M. Susstrunk ajoute qu'aussi le glacier inférieur de l'Aar présente éventuellement deux couches de glace séparées par une moraine intermédiaire et rapproche ce fait d'une description de Altmans, dans laquelle le glacier apparait retiré, laissant une grande vallée recouverte de moraines qui, depuis, ont été recouvertes par la glace.

Le fait est que, à peu près dans la zone en question, les sismogrammes obtenus par les sondages sismiques montrent deux arrivées d'ondes réfléchies, dont l'élaboration foumit deux horizons de réflexions presque parallèles. Ce problème a soulevé beaucoup de discussions parmi les glaciologues suisses. D'une part, on a considéré que la couche inférieure serait représentée par une moraine (et par conséquence, à l'aide des sondages sismiques on devait pouvoir obtenir des indications coneernant le lieu de retraite maximum des glaciers au moyen âge), mais malheureusement, d'autre part, il nous semble peu probable que des ondes réftéchies dans la surface $d u$ bedrock puissent être observées utilement après avoir pénétré deux fois une moraine de fond d'épaisseur consicérable. Il ne reste qu'à soupçonner que la deuxième réflexion soit celle causẻe normalement dans la discontinuité glace/rocher et que la première soit produite dans une intersurface dans la glace, formée par exemple par une moraine intermédiaire entre des masses de glace d'origines différentes. Malheureusement on ne sait que très peu de chose par rapport aux conditions nécessaires dans un élément miroitant pour donner lieu à une réflexion observable. Peut-être est-ce une moraine d'épaisseur inférieure à 5 ou 10 mètres qui suffit à réfléchir une certaine quantité de l'énergie d’une onde élastique longitudinale ou même est-ce déjà une zone mince d'un mélange irrégulier de débris et de la glace.

M. le Présidcnt rapproche ce phénomène de celui qui pourrait se produire aux Evettes si la calotte glaciaire du plateau à très faible pente venait à être recouverte par une extension de glace provenant de la région du col Francezetti.

M. Colder remarque que la moraine empêche la soudure des deux couches de glace.

M. Wromann trouve remarquable que le glacier qui progresse n'arrache pas cette moraine.

M. Cherrey rappelle qu'en France le maximum de la poussée glaciaire est situé entre les années 1600 et 1700 (Movar indique 1640 pour les glaciers de Chamonix). Si, actuellement, le retrait des glaciers laisse à découvert une masse glaciaire enterrée, on est forcé d'admettre qu'elle date d'une époque antérieure an mouvement d'avance des glaciers qui se situe entre le moyen âge et le $x \vee r r^{\circ}$ siècle. Ces lentilles de glace auraient été enterrées à une époque de recul glaciair'e, vraisemblablement en liaison avec les grands phénomènes des glaciations classiques. Des observations précises seraient évidemment très intéressantes.

M. le Président signale pour fixer les idées que la srande moraine frontale du glacier des Evettes a été datée 1818-1820. Elle marque la progression maxima du glacier au $x^{0}{ }^{\theta}$ siècle. Il est possible qu'au $x^{\prime} I^{0}$ siècle le même glacier ait rempli toute la cuvette des Evettes, et çue le front du glacier ait atteint à cette époque l'emplacement du refuge des Evettes.

M. le Président remercie encore une foís $M$. SüssTRUNK et le félicite pour les sondages intensifs exécutés cn Suisse, notamment au Gorner et à Finsteraar, qui ont abouti a l'élaboration de cartes sous-glaciaires, ce qui correspond à un stade d'études glaciologiques non encore atteint en France.

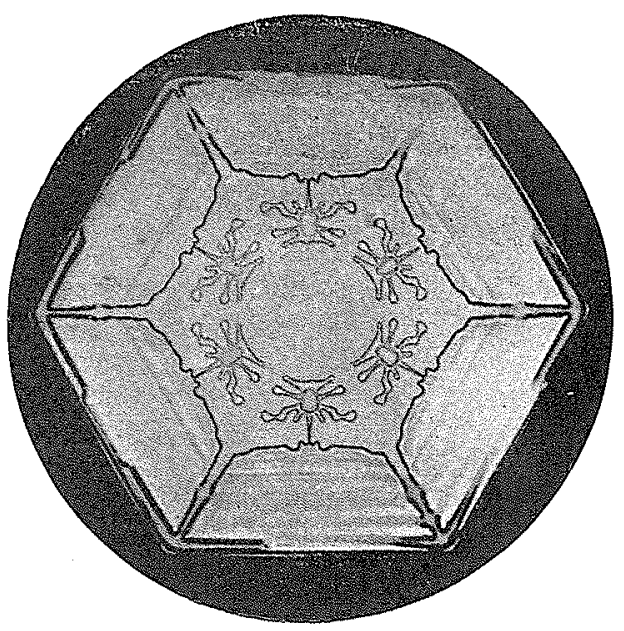

